\title{
résolution par la méthode des caractéristiques des contraintes du problème de butée dans un sable décrit par le modèle " état critique »
}

\author{
J.-M. HOVAN \\ Candidat au doctorat, département de génie civil, \\ Université du Minnesota * \\ J.GRAHAM \\ Professeur, département de génie civil, \\ Université du Manitoba *.
}

\section{Résumé}

On a résolu par la méthode des caractéristiques des contraintes, le problème bidimensionnel du mur en butée contre du sable; ce sable est défini par le modèle d'état critique. Le modèle tient compte du niveau des contraintes, de la densité de mise en place, de la compressibilité du sable et du passage d'un comportement d'écrouissage à un comportement d'antiécrouissage qui accompagne les niveaux de contraintes les plus élevés.

Les résultats font apparaître une relation linéaire entre $\log \left(k_{p}\right)$ et log (hauteur du mur) ; cette relation dépend des données du sable utilisées. Les valeurs obtenues ont été comparées avec les résultats disponibles d'essais de maquettes et de murs en vraie grandeur. Bien que les données expérimentales soient insuffisan. tes pour une application satisfaisante du modèle d'état critique, la tendance des résultats est encourageante. Plus de vérifications expérimentales sont toutefois nécessaires.

\footnotetext{
Abstract

A solution has been obtained for the scale effect of plane strain failure of a passive wall in sand using stress characteristics. The sand is characterized by a critical state model that takes account of local stress levels in the failure zone; the placement density; the compressibility of the sand; and the transition from strain softening (expansive) behaviour to strain hardening (compressive) beha: viour with increasing stresses. The results suggest linear relationships between $\log \left(K_{p}\right)$ and $\log$ (wall height) depending on the properties of the sand. Calculated results have been compared with available published results from models and large scale walls. Although the published data does not permit development of the corresponding critical state models, the trend of the calculated results is encouraging.
}

- Minneapolis, USA 55414.

* Winnipeg, Canada R3T 2N2. 


\section{INTRODUCTION}

La stabilité des murs en butée contre du sable est habituellement estimée à partir de calculs d'équilibre limite qui tiennent compte des forces de poids propre dans la zone de rupture. Les forces de rupture sont d'ordinaire exprimées à l'aide du coefficient de butée $\mathrm{K}_{p}$, par la formule $\mathrm{P}_{p}=0,5 \mathrm{~K}_{p} \gamma \mathrm{H}$ (les notations sont résumées à la fin de larticle). La plupart des solutions existantes utilisent des surfaces potentielles de rupture composées de spirales logarithmiques et de lignes droites (BIAREZ et al., 1965). De telles solutions impliquent des restrictions sévères sur les conditions de frontière et de domaine qui peuvent être résolues. D'autre part, la plupart des analy. ses exigent que l'angle de frottement sol-structure soit constant alors qu'il est en fait fonction des déplacements et n'est pas une propriété inhérente du sol (JAMES et BRANSBY, 1970 ; MORGENSTERN et EISENSTEIN, 1970).

Dans le présent contexte, il est aussi important de remarquer qu'on suppose habituellement que la résistance d'un sable mis en place avec une densité uniforme est gouvernée par la contrainte limite de cisaillement, et par le critère de Mohr-Coulomb $\tau_{f}=\sigma_{n} \tan \Phi$ où l'angle de résistance au cisaillement reste constant (fig. 1a). (Tout au long de notre étude, nous utilisons les contraintes effectives et la convention habituelle " ' qui les représente a été supprimée pour raison de simplicité.)

En réalité, l'enveloppe de rupture n'est pas droite mais courbe ; aussi $\Phi$ n'est-il pas constant. La courbure résulte de l'effet du niveau de pression sur l'importance relative des trois composantes de la résistance du cisaillement des sables, à savoir (1) le frottement de la matière minérale au contact des grains (2) l'enchevêtrement des grains, et (3) la dilatance (ROWE, 1969). La figure 1b présente une relation entre $\Phi$ et la pression (LADANYI, 1960 ; DE BEER, 1970). En pratique, les ingénieurs de mécanique des sols tiennent compte de la courbure de l'enveloppe de rupture lorsqu'ils utilisent des valeurs de $\Phi$ variant en fonction de la taille de l'ouvrage (TCHENG et ABSI, 1969 ; LAPERCHE, 1976) et de la densité relative initiale du sable (par exemple, BJERRUM, 1973). Bien qu'il soit possible d'arriver à des valeurs comparables entre les résultats d'essais de maquettes de laboratoire et des calculs de butée (GRAHAM, 1971), de telles valeurs sont trop grandes pour être utilisées en pratique (JAMES et BRANSBY, 1970). Par ailleurs, le comportement des maquettes de mur de laboratoire est beaucoup plus « cassant s que celui observé pour des ouvrages en vraie grandeur. Ces deux effets ont conduit les ingénieurs à traiter les valeurs analytiques et les valeurs de laboratoire avec scepticisme.

Les changements du comportement contraintedéformation du sable en fonction de la densité relative sont bien connus pour les essais triaxiaux (fig. 2). Les sables denses se dilatent lors du cisaillement alors que les sables lâches se rétractent. Des changements similaires de comportement se produisent lorsque les niveaux de contrainte augmentent : les sables de même densité relative initiale se dilatent sous faible contrainte alors qu'ils se rétractent sous forte contrainte lors du cisaillement. Les résultats de la figure 2 ont été normalisés en divisant la contrainte par la pression de confinement $\sigma_{\text {cell }}$ et les termes \& écrouissage * et * antiécrouissage * remplacent les termes habituels de mécanique des sols * lâche " et « dense ", ou « faible pression " et "grande pression ".

Dès lors, le problème consiste à construire un meilleur modèle de comportement du sable et à l'introduire dans une analyse plus complète. MANDEL (1943) avait présenté les équations générales de Kötter pour un matériau homogène à courbe intrinsèque quelconque (SALENÇON, 1974). Le comportement d'un sol nonhomogène où la cohésion augmente linéairement avec la profondeur fût présenté par SALENÇON et al. (1976). Des calculs précédents présentés par GRAHAM (1974) utilisaient le modèle de résistance simplifié (fig. 1b) de DE BEER (1970) d'après LADANYI (1960), selon lequel $\Phi$ variait en fonction du logarithme de la contrainte principale moyenne. Dans leur étude, la plage de variation de $\Phi$ dépendait de la densité de mise en place du sable. On utilise ici un modèle de sable décrit dans Critical State Soil Mechanics d'ATKINSON et
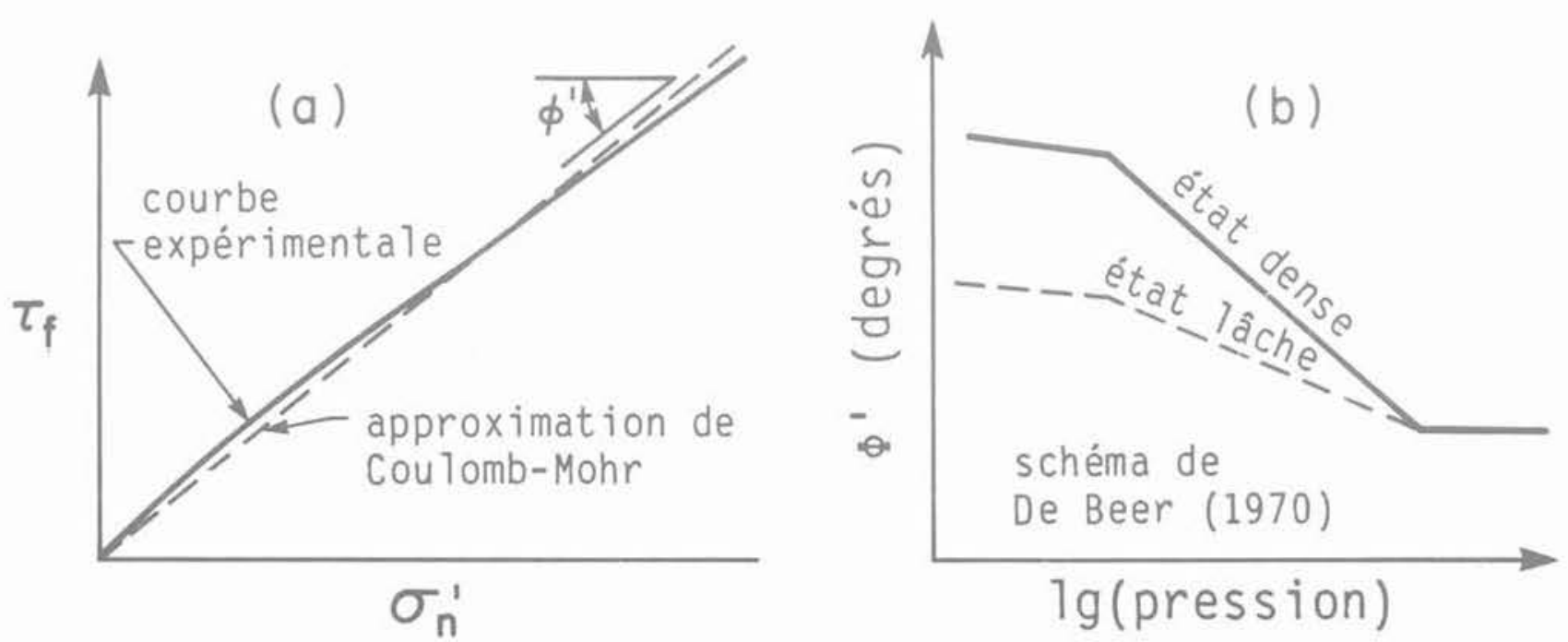

Fig. 1 - (A) Approximation de Coulomb-Mohr pour l'enveloppe de rupture.

(B) Relation $\Phi^{\prime}-\lg$ (pression) (d'après DE BEER, 1970). 
BRANSBY (1978). Ce modèle est incorporé dans la méthode des caractéristiques de contraintes pour résoudre la rupture des murs en butée. Nous prenons aussi en compte les effets de la compressibilité du sable, ce qui permet une première analyse théorique de l'influence de la hauteur d'un mur sur les valeurs des contraintes de rupture du sable.

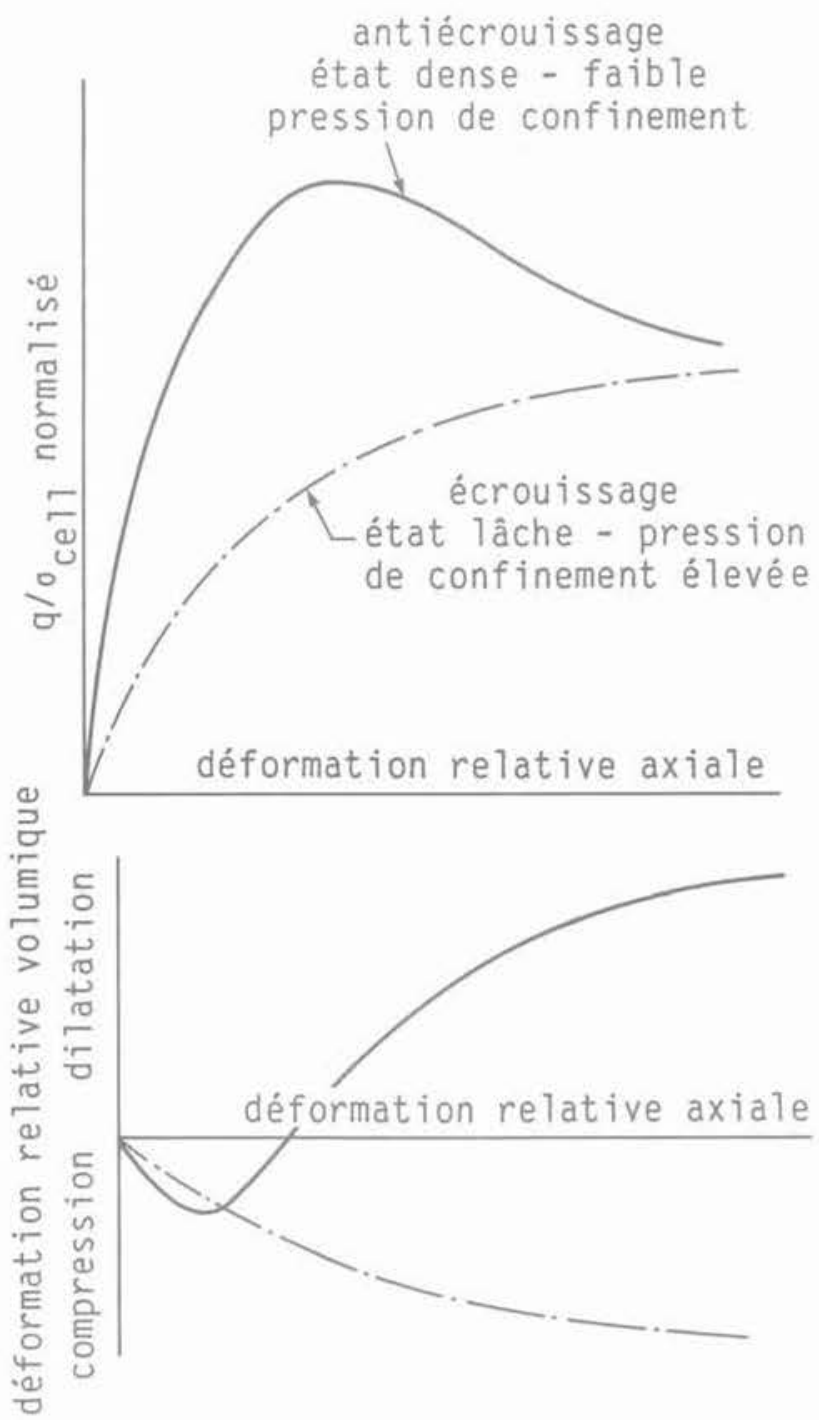

Fig. 2 - Comportement contrainte-déformation et déformation volumique du sable.

\section{LE MODELE D'ETAT CRITIQUE}

* L'état critique » a été bien développé pour les argiles remaniées (ROSCOE et BURLAND, 1968) et a conduit à des modélisations utiles des propriétés des argiles naturelles (GRAHAM et al., 1983). Le modèle définit des lignes parallèles de pente $\lambda$ dans l'espace $V$-log(p) pour la consolidation et la résistance au cisaillement d'un échantillon normalement consolidé. Il prend aussi en compte une grande partie du comportement plus complexe de la surconsolidation. La pente $\lambda$ est appelée ici * compressibilité ».

Pour les sables, un modèle similaire n'a pas encore été mis au point, bien que des données expérimentales existent (fig. 3a) et qu'elles aient été récemment confirmées par des essais triaxiaux sous conditions non-drainées sur des sables de la mer de Beaufort canadienne (fig. 3b ; BEEN et al., 1985).
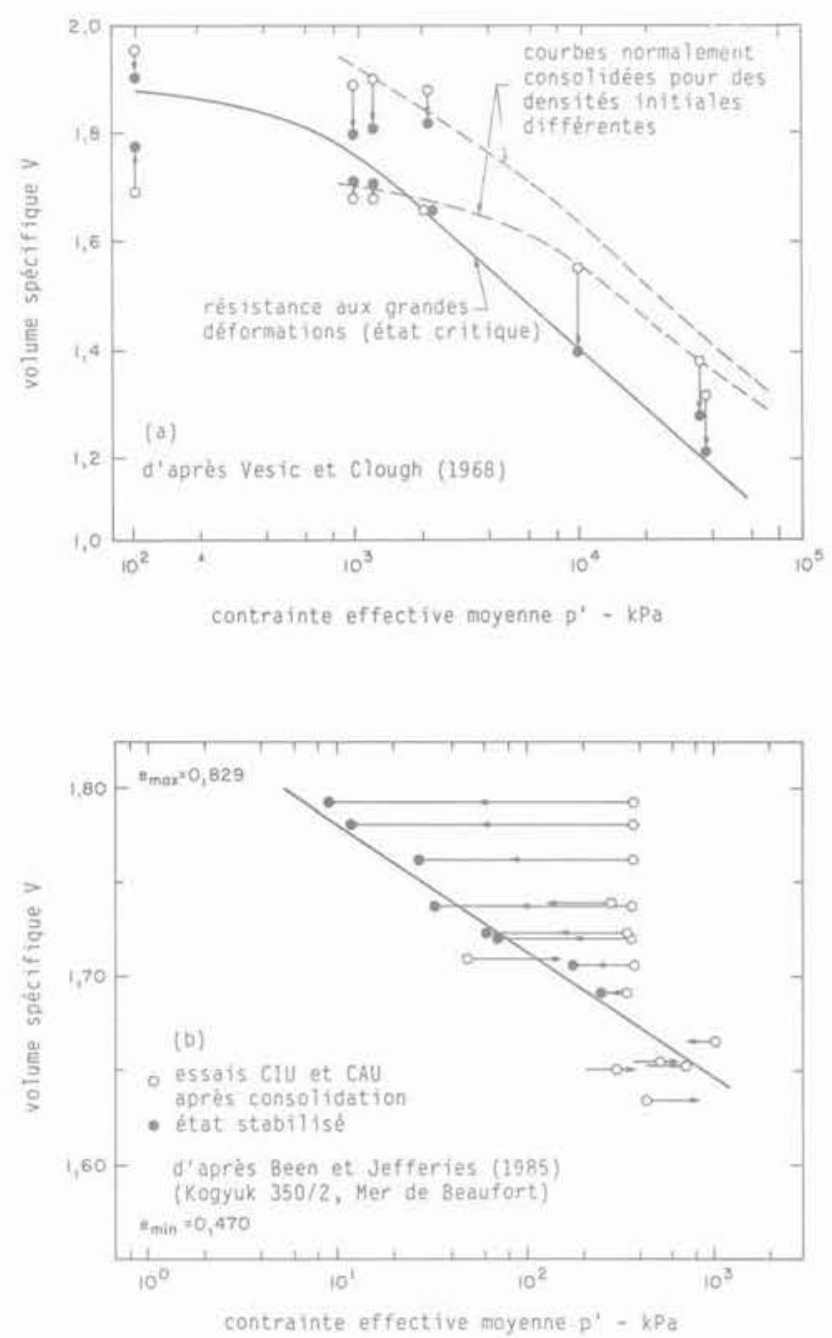

Fig. 3 - Données d'état critique des essais triaxiaux pour des sables.

ATKINSON et BRANSBY (1978) proposent une nouvelle méthode graphique (fig. 4a) pour décrire l'espace $\mathrm{p}, \mathrm{q}, \mathrm{V}$. On projette l'espace $\mathrm{p}-\mathrm{V}$ sur un plan constant $\mathrm{p}=1 \mathrm{kPa}$ dans la direction $\lambda$ et une nouvelle variable $V_{\lambda}=V+\lambda_{\lambda} \ln (\mathrm{p})$ est introduite. La relation entre la contrainte et la déformation volumique d'un essai peut être alors tracée graphiquement avec en ordonnée le rapport des contraintes $q / p$ et en abcisse, le paramètre de pression-volume $V_{\lambda}$ (fig. $4 \mathrm{~b}$ ). La section supérieure de la surface de l'état limite peut être alors approchée par l'enveloppe droite $A B$ de la figure $4 \mathrm{~b}$. Cette droite décrit les contraintes de rupture associées à l'anti-écrouissage 

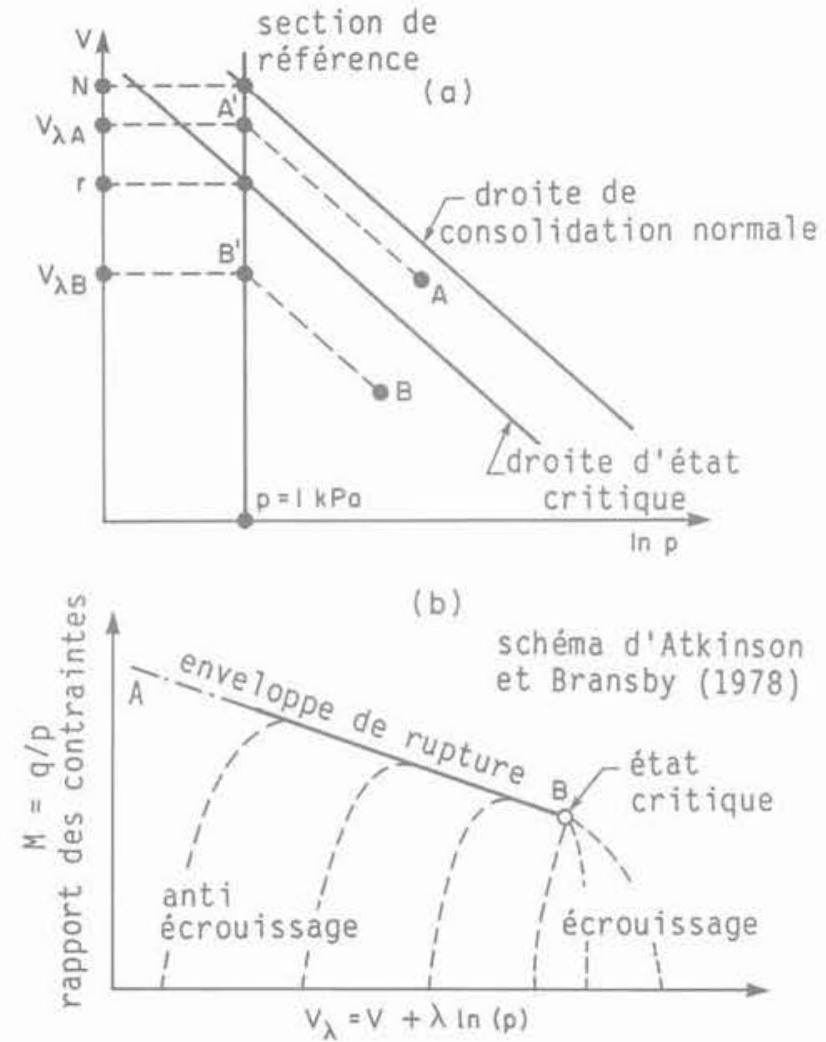

Fig. 4 - Modèle d'état critique pour les sables.

d'un échantillon " dense * ou « faible pression " de la figure 2. Le point B correspond à la rupture d'écrouissage normalisé d'un échantillon "lâche "ou " haute pression ». Ce point correspond aussi à l'état critique.
Le volume reste constant et l'état critique est atteint par écrouissage après grande déformation de l'échantillon. Prendre comme nouvelle variable $V_{\lambda}=V+\lambda \ln (p)$ dans le modèle du sol, permet de tenir compte de la courbure de l'enveloppe expérimentale de rupture (fig. 1a) en utilisant le volume spécifique de mise en place $V_{0}$ et la compressibilité $\lambda$ du sable. On peut noter que $\mathrm{M}=(\mathrm{q} / \mathrm{p})$ est lié à l'angle $\Phi(\Phi$ dépendant de la pression locale p) par le relation $\sin \Phi=3 \mathrm{M}(6+\mathrm{M})$. Avec ce modèle, il n'est plus possible de décrire un sable simplement par son angle $\Phi$, car $\Phi$ dépend désormais du niveau de pression, de la densité de mise en place et de la compressibilité $\lambda$. Définir la résistance de cette façon en fonction de la pression permet d'étudier la variation des coefficients de butée en fonction de la hauteur du mur. Par comparaison, les analyses basées sur le modèle plus simple $\Phi=$ constante prédisent que le coefficient de butée $\mathrm{K}_{p}(\Phi)=2 \mathrm{P}_{p} / \gamma \mathrm{H}^{2}$ est indépendant de la hauteur du mur $\mathrm{H}$. On peut noter que le modèle du sol de la figure 4 b présente une limite en B qui correspond à $\Phi_{\min }$ où l'enveloppe de rupture devient droite dans les diagrammes $q-p$ et $V$ - $\ln (p)$ (fig. 3 ).

Les calculs des coefficients de butée ont été effectués pour l'ensemble des valeurs données dans le tableau 1. On a séparé l'analyse en deux groupes, un premier où la plage de variation des paramètres est étroite, un deuxième où ces mêmes variations sont plus importantes. Dans chaque groupe, quatre valeurs de $\lambda$ ont été testées. Dans le cas de la plage de variation étroite, les propriétés du sable ne sont affectées que faiblement par les changements de pression alors que dans celui de la plage de variation large, de plus grandes variations sont enregistrées. Les paramètres du sol du tableau 1 sont représentés par les deux séries d'enveloppes $q / p-V_{\lambda}$ des figures $5 \mathrm{a}$, b correspondant respectivement aux plages de variations étroite et large. (a) plage de variation étroite

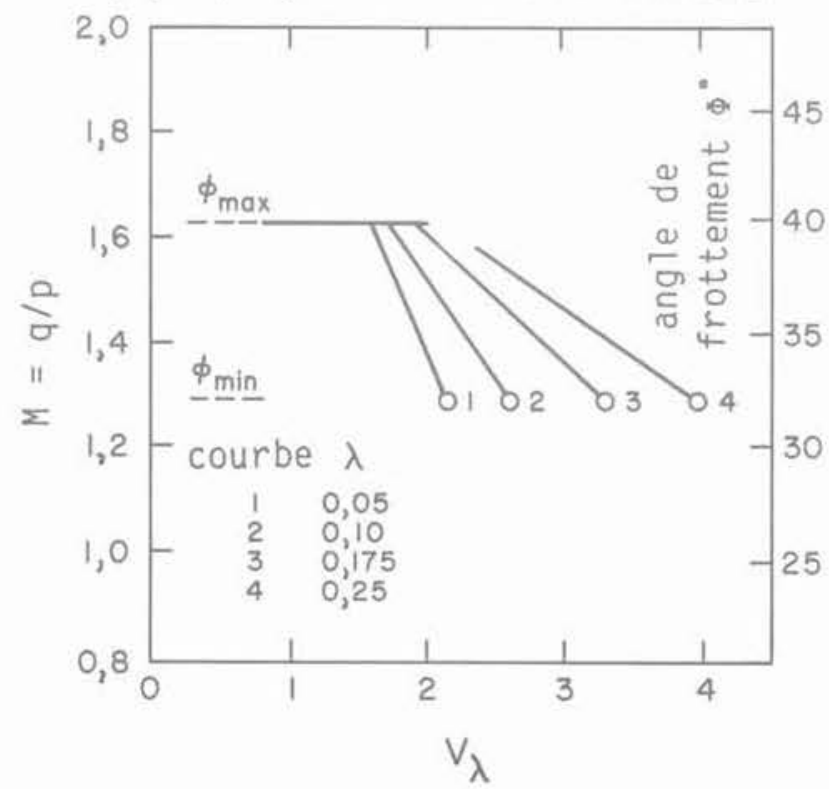

(b) plage de variation large

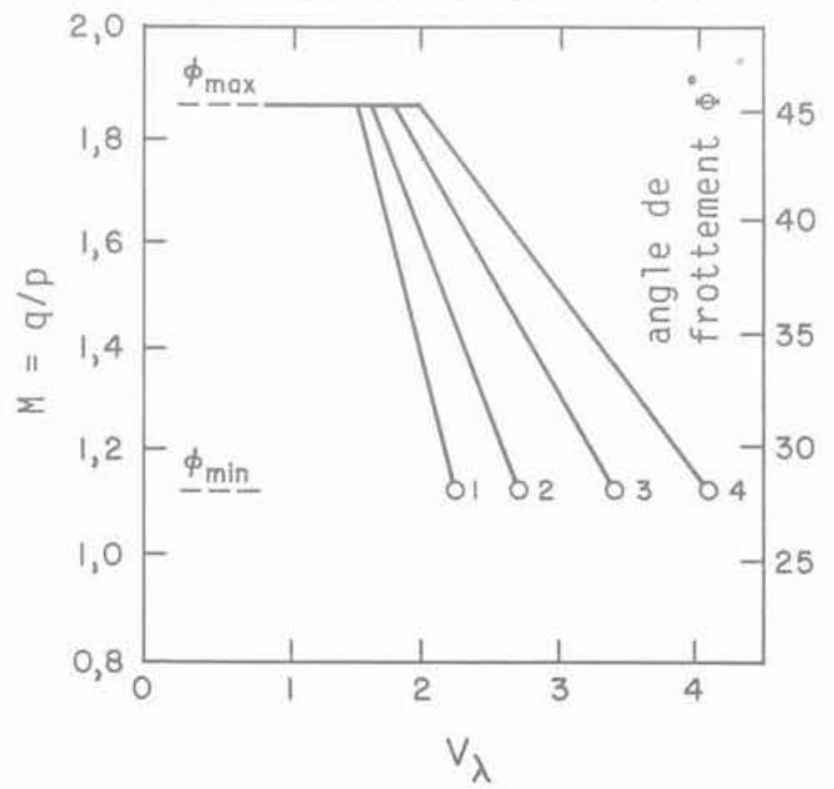

Fig. 5 - Enveloppes de rupture utilisées : (a) plage de variation étroite (b) plage de variation large. 
Tableau 1

PARAMETRES DU SABLE POUR MODELISER LES ENVELOPPES DE RUPTURE DE LA FIGURE 5

\begin{tabular}{|l|c|c|c|c|}
\hline & \multicolumn{2}{|c|}{$\begin{array}{c}\text { Plage } \\
\text { de variation } \\
\text { étroite }\end{array}$} & \multicolumn{2}{|c|}{$\begin{array}{c}\text { Plage } \\
\text { de variation } \\
\text { large }\end{array}$} \\
\cline { 2 - 5 } $\begin{array}{l}\text { Valeurs de } p(\mathrm{kPa}) \\
\text { Volume spécifique }\end{array}$ & 10 & $10^{4}$ & 10 & $10^{4}$ \\
$\mathrm{~V}=(1+\mathrm{e})$ & 1,5 & 1,7 & 1,4 & 1,8 \\
$\Phi_{\min }, \Phi_{\max }$ (degrés) & 32 & 40 & 28 & 45 \\
\hline
\end{tabular}

Notes :

(1) $(\mathrm{V})_{i}=\mathrm{V}_{i}+\lambda \ln \mathrm{p}$

(2) Valeurs choisies de $\lambda=0,05-0,10-0,175-0,25$

\section{APERÇU DE L'ANALYSE}

La méthode des caractéristiques de contraintes a été décrite en détail par SOKOLOVSKII (1965), GRAHAM (1968) et SALENCON (1974) et ne sera revue que brièvement ici. En déformation plane, on associe les équations d'équilibre :

$$
\frac{\partial \sigma_{\mathrm{Zz}}}{\partial \mathrm{z}}+\frac{\partial \sigma_{\mathrm{Xz}}}{\partial \mathrm{x}}=\gamma ; \frac{\partial \sigma_{\mathrm{Xx}}}{\partial \mathrm{x}}+\frac{\partial \sigma_{\mathrm{Zx}}}{\partial \mathrm{z}}=0
$$

avec le critère de rupture de Mohr-Coulomb,

$$
\max \left(\left|\tau_{n}\right|-f\left(\sigma_{n}\right)\right)=0
$$

qui peut être écrit :

$$
\begin{gathered}
\frac{\partial}{\partial \omega}\left(\left|\tau_{n}\right|-f\left(\sigma_{n}\right)\right)=0 \\
\text { ou } \sigma_{n}=\left\{\sigma_{1}+\sigma_{3}\right) / 2+\left\{\left(\sigma_{1}-\sigma_{3}\right) / 2\right\} \cos 2 \omega \\
\tau_{n}=\left\{\left(\sigma_{1}-\sigma_{3}\right) / 2\right\} \sin -2 \omega
\end{gathered}
$$

La figure 6a est une représentation schématique de ces expressions mathématiques. En utilisant les équations (3) et (4), on arrive à la condition générale $\operatorname{cotg}(2 \mu)=f^{\prime}\left(\sigma_{n}\right)$ où $2 \mu=\pi-2 \omega$. Quand on suppose $\Phi$ constant, on a alors $\mathrm{f}\left(\sigma_{n}\right)=\sigma_{n} \operatorname{tg}(\Phi)$ et $\mu=\pi / 4-\Phi / 2$. L'angle $\mu=\pi / 4-f^{\prime}\left(\sigma_{n}\right) / 2$ détermine la direction des plans de cisaillement limite (lignes de glissement) en un point $P$ (figure $6 \mathrm{~b}$ ), en fonction des états locaux de contraintes et de la pente locale $f^{\prime}\left(\sigma_{n}\right)$ de l'enveloppe de rupture courbe (ce que GRAHAM et POLLOCK (1972) ont appelé « la valeur locale $\Phi$, fonction de la pression $»$ ).

Après avoir d'abord transformé les coordonnées du tenseur des contraintes au point $\mathrm{P}$ dans les axes $\mathrm{x}$ et $\mathrm{z}$ en grandeur sans dimension (fig. $6 \mathrm{~b}$ ), puis substitué l'équation (4) dans l'équation (1) et enfin, introduit des transformations logarithmiques pour séparer les produits résultants, on obtient deux familles de caractéristiques de contraintes réelles.

$$
\mathrm{dn} / \mathrm{dz}=\mathrm{a},
$$

dans la direction de glissement

$$
\mathrm{dx} / \mathrm{dz}=\operatorname{tg}(\psi-\mu)
$$

dans la direction conjuguée

$$
\text { et } d \xi / d z=b \text {, }
$$

$$
\mathrm{dx} / \mathrm{dz}=\operatorname{tg}(\psi+\mu) ; \text { où }
$$
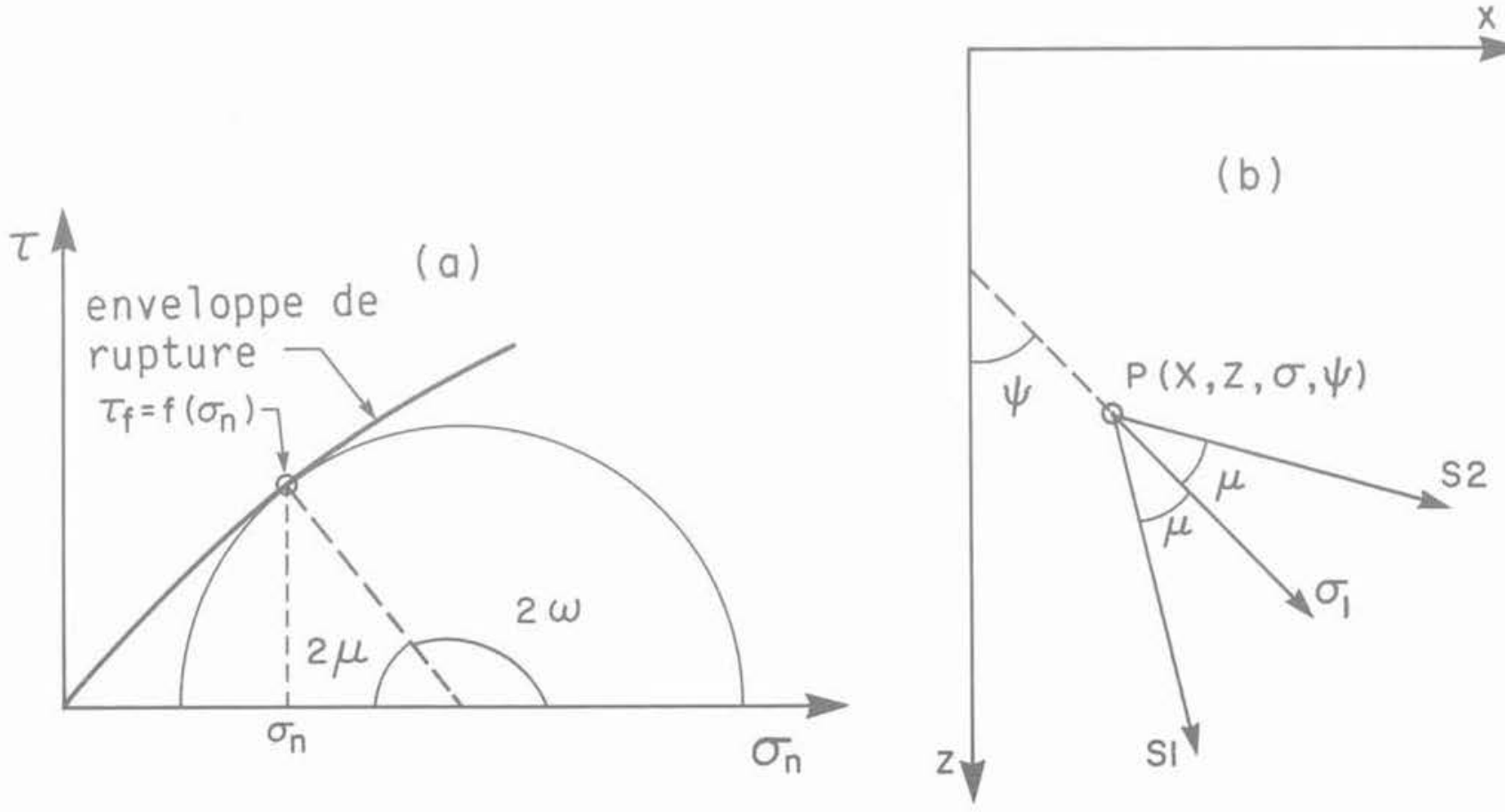

Fig. 6 - (A) Condition de rupture d'après Coulomb-Mohr, enveloppe non linéaire (B) Définition des variables. 


$$
\begin{gathered}
\mathrm{a}, \mathrm{b}=\frac{ \pm 1}{2 \sigma \sin \Phi} \cdot \frac{\sin (\psi \pm \mu)}{\cos (\psi \pm \mu)} ; \sigma=\left(\sigma_{1}+\sigma_{3}\right) / 2 \\
\eta, \xi=\ln (\sigma) / 2 \operatorname{tg} \Phi \pm \mu
\end{gathered}
$$

Ce système de quatre équations à quatre inconnus (x, z, $\sigma, \psi)$ peut être résolu pour tout nouveau point d'une masse de sol en rupture, à partir d'un point frontière ou à partir de tout autre point du domaine déjà calculé dans les équations (5) et (6).

\section{Tableau II}

ORGANIGRAMME DU CALCUL D'UN NOUVEAU POINT $(x, z, \sigma, \psi)(\Phi)$

A PARTIR DE DEUX AUTRES POINTS DEJA CONNUS $(x, z, \sigma, \psi)_{1,2}$

Entrée des données $(x, z, \sigma, \psi)_{1,2}$

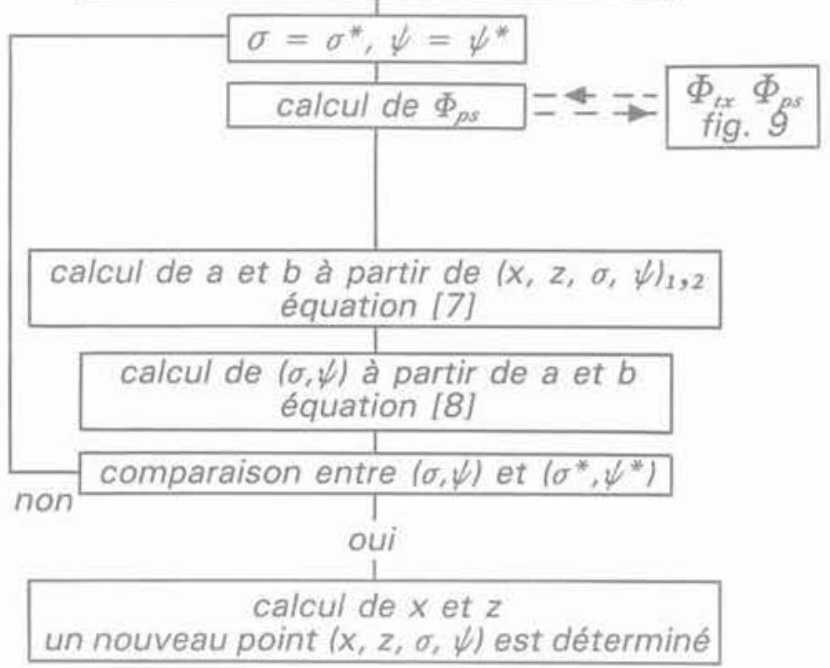

Aucune solution analytique n'est disponible, et on utilise la méthode de différences finies. Celles-ci prend dans les équations (5) et (6) les variations de la direction de la contrainte principale (SOKOLOVSKII, 1965) ; de la contrainte principale moyenne (GRAHAM, 1968) ; et de l'angle de cisaillement local $\Phi$ (GRAHAM et POLLOCK, 1972) ; HOVAN 1985). Le tableau II présente un organigramme des calculs nécessaires.

\subsection{Conditions aux limites}

Le problème du mur en butée fut considéré par HAJAL (1961) et SALENÇON (1972). Le mur de la figure 7 est résolu avec les hypothèses suivantes :

a) Mur de hauteur $H$ retenant un remblai de sable sans cohésion, de poids volumique $\gamma$, de compressibilité $\lambda$, et de résistance définie par un des modèles d'état critique de la figure 6.

b) Frontière inférieure d'une zone massive de Rankine uniquement définie pour un remblai horizontal et sans surcharge. On peut noter que lorsque $\Phi=\left(\sigma_{n}\right)$ comme le montre la figure 1a, le bord de la zone passive n'est pas droit mais s'infléchit vers le bas.

c) Singularité au coin supérieur du mur, là où les lignes de glissement s'écartent en éventail avec des pentes différentes $\left(\psi_{i}-\mu\right)$ et où $\sigma_{i} / \sigma_{O}=\exp \left\{2\left(\psi_{i}-\psi_{0}\right) \operatorname{tg} \Phi_{i}\right\}$.

d) Mur complètement rugueux où $\delta=\Phi\left(\sigma_{n}\right)$. Pour simplifier les comparaisons avec des solutions existantes, on ne fait pas varier ici la valeur de l'angle de frottement mobilisé en fonction de la profondeur, comme le sug. gèrent JAMES et BRANSBY (1970) et GRAHAM (1971).

La figure 7 présente une solution typique obtenue par la méthode des caractéristiques de contraintes. La condition de non-surcharge du remblai entraîne des difficultés de calculs à la singularité (c) (KRAVTCHENKO

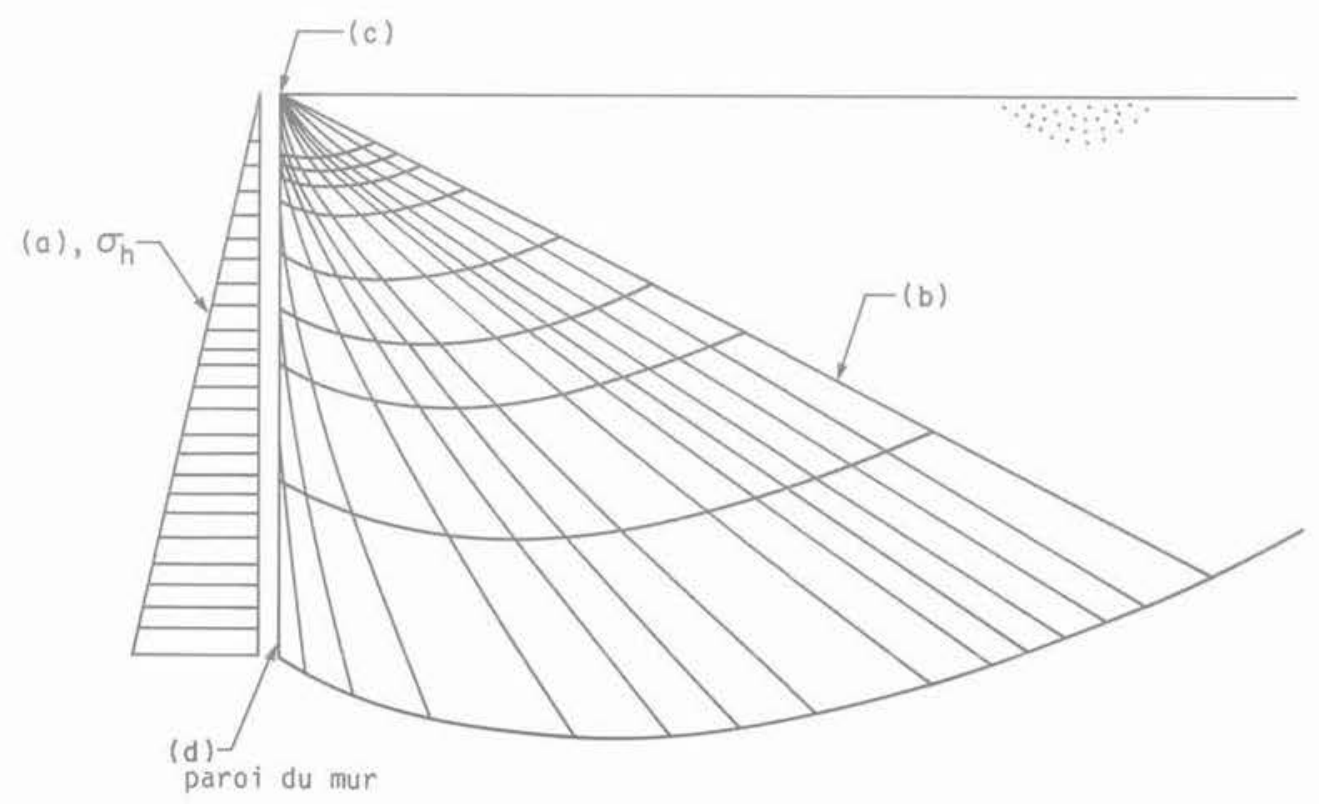

Fig. 7 - Solution typique des caractéristiques de contraintes pour le mur en butée. 
et SIBILLE, 1965) et il faut recourir à un calcul de différences finies pour évaluer les fonctions logarithmiques $\xi$ et $\eta$, indéterminées pour $\sigma=0$. Ces difficultés peuvent être résolues (GRAHAM. 1968) en commençant initialement avec une surcharge sur la surface (b). On calcule la zone de rupture correspondante, puis on contracte vers le point (c) cette zone de rupture. La contraction est en fait un changement d'échelle par un facteur arbitraire $(x 10)$, dont le but est de diminuer l'influence de la surcharge. La surface réduite obtenue est alors utilisée comme spirale de départ et permet de recalculer le domaine de contraintes jusqu'à la zone qui se trouve sous l'influence du chargement. A la fin de cette deuxième phase, les effets de la surcharge ont été réduits par le facteur de contraction. On répète alors le procédé jusqu'à ce que les changements d'échelle ne produisent plus que de petites variations acceptables de $\mathrm{K}_{p}$.

GRAHAM (1974) a montré que, pour des conditions aux limites identiques, on obtient des valeurs de $\mathrm{K}_{p}$ tout à fait similaires par la méthode des surfaces potentielles de rupture et par la méthode des caractéristiques de contraintes. La seconde méthode a l'avantage de permettre la modélisation de distributions de frottement aux frontières plus réalistes (JAMES et BRANSBY, 1970 ; GRAHAM, 1971) ainsi que la prise en compte de données plus complexes dans la zone de rupture, comme ici le modèle d'état critique présenté aux figures 4 et 5 . La validité du nouveau programme d'ordinateur a été vérifiée (tableau III) en comparant les valeurs de la solution de GRAHAM (1971) ( $\Phi$ reste constant) avec celles produites par une solution qui utilise une version simplifiée de la figure 5; dans ce cas, on spécifie $\Phi_{\min }=\Phi_{\max }$ afin d'avoir $\Phi$ constant. Les différences entre les deux solutions sont négligeables.

Tableau III

COMPARAISON DES VALEURS DE K D'APRES UNE SOLUTION $\Phi=$ CONSTANTE

\begin{tabular}{|l|c|l|l|}
\hline$\Phi$ (degrés) & 30 & 34 & 38 \\
$\mathrm{~K}_{p}$ (Auteurs) & 5,65 & 7,85 & 11,60 \\
$\mathrm{~K}_{p}$ (Graham, 1971) & 5,6 & 7,8 & 11,3 \\
\hline
\end{tabular}

\subsection{Résistance en déformation plane}

La relation $\mathrm{q} / \mathrm{p}(\Phi)$ fonction de $V_{\lambda}$ de la figure 5 provient habituellement d'essais de laboratoire et correspond alors à des valeurs triaxiales $\Phi_{t x}$. Cependant, l'analyse présentée à l'organigramme du tableau II et le domaine des caractéristiques de contraintes de la figure 7 correspondent à des conditions de déformation plane, et font appel à des valeurs $\Phi_{p s}$. GRAHAM (1971) a montré qu'il est nécessaire d'utiliser l'angle $\Phi_{p s}$, mobilisé au niveau de contraintes adéquat, pour comparer les valeurs théoriques et les valeurs expérimentales issues d'essais de maquettes de laboratoire.

Il est bien connu que les angles de résistance au cisaillement en déformation plane $\Phi_{p s}$ sont plus grands que les valeurs $\Phi_{t x}$ correspondantes obtenues à partir d'essais triaxiaux symétriques. La figure 8 montre trois relations entre $\Phi_{p s}$ et $\Phi_{t x}$ provenant de publications. Pour notre étude de mur en butée, la relation de Bishop (1966) est utilisée sous la forme suivante:

(i) pour $\Phi_{t x} \leq 33^{\circ}, \Phi_{p s}=\Phi_{t x}$

(ii) pour $33^{\circ} \leq \Phi_{t x} 36^{\circ}$, $\ln \Phi_{p s}=1,666 \ln \Phi_{t x}-2,336$

(iii) pour $36^{\circ} \leq \Phi_{x}, \ln \Phi_{p s}=1,293 \ln \Phi_{t x}-1,002$

Le passage d'un type de valeurs à un autre est effectué par un sous-programme (tableau II) qui évalue la valeur $\Phi_{p s}$ nécessaire pour le calcul d'un nouveau point du domaine des caractéristiques de contraintes. On opère en termes de contraintes locales et de la compressibilité de la figure 5.

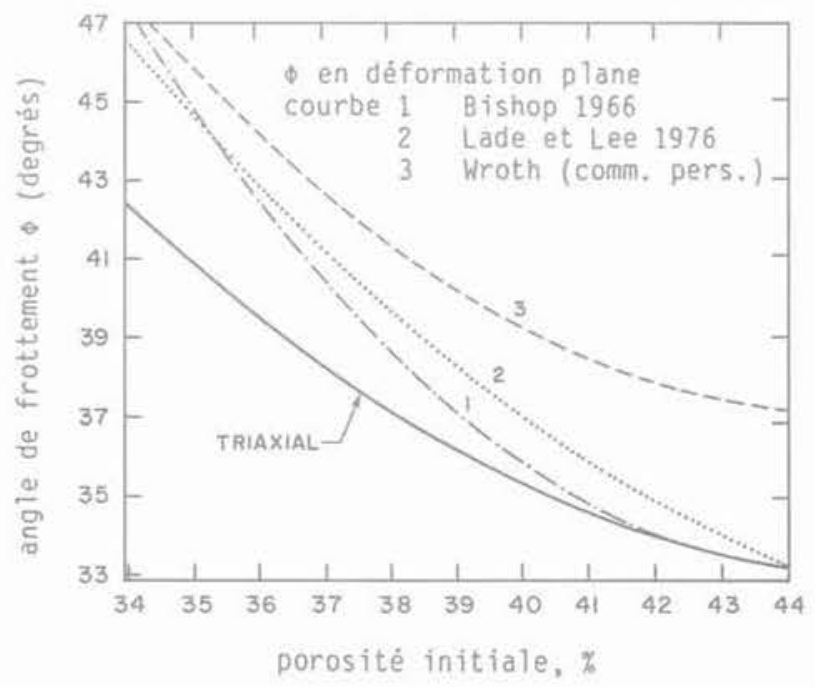

Fig. 8 - Relations expérimentales entre les angles de frottement en déformation plane et en déformation triaxiale.

\section{RESULTATS}

La force de rupture du mur $\mathrm{P}_{p}$ est l'intégrale $\int \sigma_{x} \mathrm{~d} z$, prise le long de la partie arrière du mur (d) de la figure 7 . Les valeurs de $\sigma_{x}$ augmentent avec la hauteur du mur, mais la variation n'est pas linéaire quand l'enveloppe de rupture est courbe comme dans la figure la. Toutefois, pour simplifier les comparaisons, il s'avère pratique d'utiliser la définition usuelle $\mathrm{K}_{p}=2 \mathrm{P}_{p} / \gamma \mathrm{H}^{2}$. Les figures 9 et 10 présentent les valeurs du coefficient de butée $\mathrm{K}_{p}$ en fonction de la hauteur du mur et de la compressibilité du sable utilisant les plages large et étroite des données du sol définies par la figure 5 . Comme la figure $4 \mathrm{~b}$ modélise la courbure de l'enveloppe de rupture, $\mathrm{K}_{p}$ varie en fonction de la hauteur du mur et ce, bien qu'il s'agisse d'un paramètre sans dimensions. Cette variation traduit les effets dus à la taille de l'ouvrage (DE BEER, 1970 ; LAPERCHE, 1976 ; HABIB, 1985 ; KIMURA et al., 1985). Un mur plus haut conduit à des contraintes plus importantes dans la zone de rupture ; il mobilise donc des valeurs de l'angle $\Phi$ plus petites, et ainsi les valeurs de $\mathrm{K}_{p}$ sont réduites. Le modèle d'état critique permet une première approche quantitative de la transition écrouissage-antiécrouissage qui est liée à la compressibilité $\lambda$. 

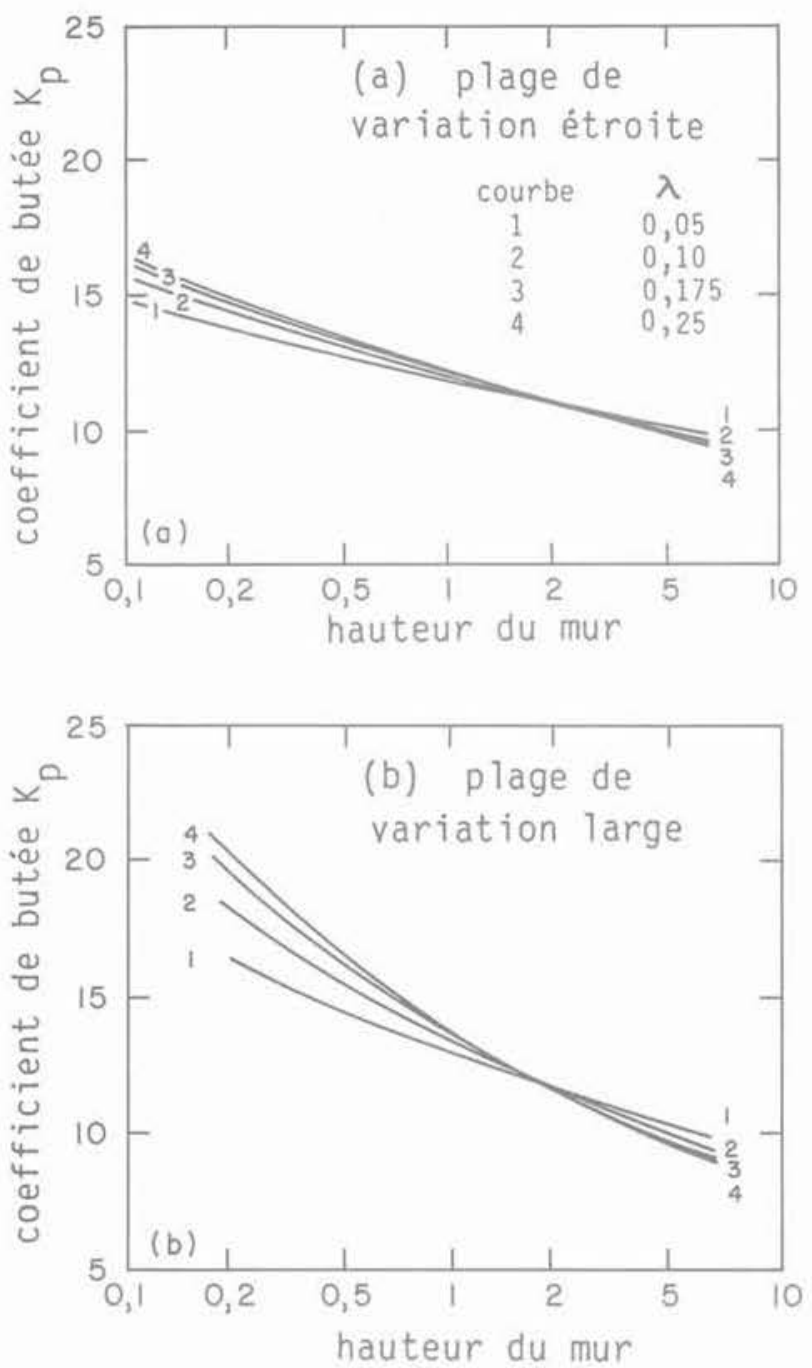

Fig. 9 - Coefficients de butée $K_{p}$ en fonction de $/ g$ (hauteur du mur)

La figure 9 présente un diagramme semi-logarithmique de $\mathrm{K}_{p}$ fonction de la hauteur $\mathrm{H}$ du mur pour quatre valeurs de $\lambda$. Neuf valeurs de hauteurs de mur ont été prises en compte (voir aussi figure 10). En accord avec la discussion qualitative précédente, $\mathrm{K}_{p}$ décrô̂t lorsque Haugmente. La plage de variation des résultats est plus grande dans le cas de la plage de variation large de données du sable, où les propriétés changent beaucoup en fonction de la pression. Elle est plus petite dans le cas de la plage de variation étroite. Il y a de plus une particularité de la figure 9 qui mérite attention. Pour de plus petits murs ( $\mathrm{H}$ petit), de petites valeurs de $\lambda$ conduisent à de plus petites valeurs de $\mathrm{K}_{p}$. Cependant, pour des murs hauts, le comportement est renversé et ce sont les plus grandes compressibilités qui produisent les plus petites valeurs de $\mathrm{K}_{p}$.

Ce comportement est encore plus évident sur la figure 10. Pour les deux plages de variations étroite et large des données du sable, les valeurs de $\mathrm{K}_{p}$ augmentent avec $\lambda$ quant la hauteur du mur est faible, mais décroissent quand cette même hauteur est grande. L'explication du phénomène est complexe et se trouve dans le modèle d'état critique de la figure 4 . On doit se rappeler que d'après cette figure, les plus grandes valeurs de $V_{\lambda}$ sont associées aux plus grandes valeurs de $\mathrm{p}$ ou aux plus grandes valeurs de $\lambda$. Pour les deux plages de variation étroite et large de l'angle $\Phi$ de la figure 5 entre $\mathrm{M}_{\max }$ et $\mathrm{M}_{\min }$, la pente de l'enveloppe de rupture par rapport à l'axe $V_{\lambda}$ décroît lorsque $\lambda$ augmente. Ainsi pour de petits murs ( $\mathrm{H}$ petit) où la plage des contraintes du sable est petite, on obtient une plage des valeurs $M(\Phi)$ plus grande pour des petites valeurs de $\lambda$ que pour des grandes valeurs de $\lambda$. Il en résulte des valeurs moyennes plus petites de l'angle mobilisé $\Phi$. Ce qui signifie que pour de petits murs, de plus petites valeurs de $\mathrm{K}_{p}$ sont associées aux valeurs de $\lambda$ plus petites.
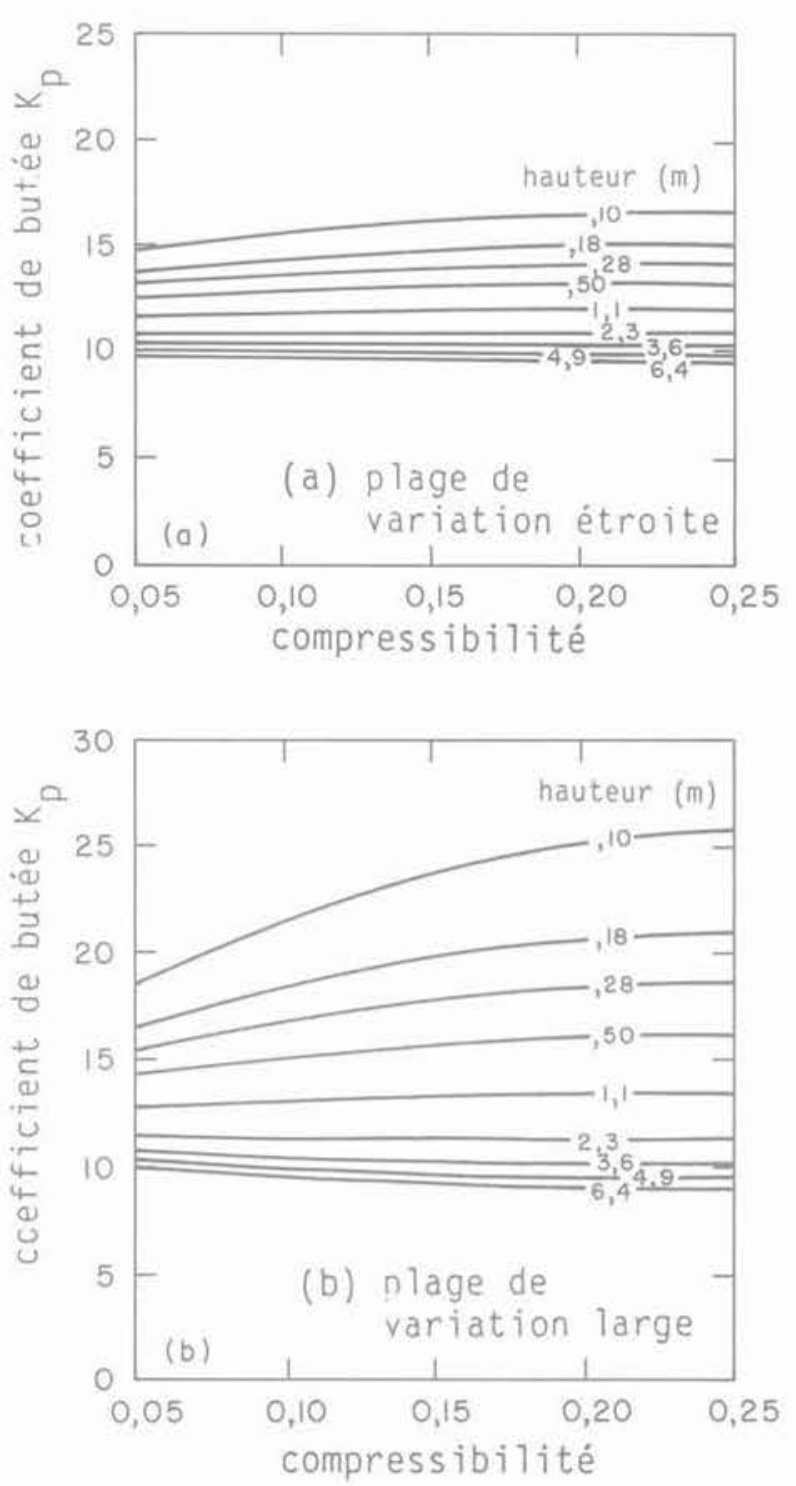

Fig. 10 - Coefficients de butée $K_{p}$ en fonction de la compressibilité $\lambda$.

La possibilité de rupture diminue lorsque le sol devient compressible, car le nombre de contacts entre particules augmente au fur et à mesure que la contrainte croît. La rupture intervient alors pour des contraintes plus élevées et donne un angle $\Phi$ mobilisé plus grand. Il en 
résulte que des valeurs des $\mathrm{K}_{p}$ élevées correspondent aux fortes valeurs de $\lambda$.

La situation est différente pour des murs hauts (H grand). Dans ce cas, les contraintes près de la limite de la zone passive (b) (fig. 7) sont très petites, et deviennent très grandes au bas de la limite (d). La distribution des valeurs mobilisées $\mathrm{q} / \mathrm{p}(\Phi)$ dépend de la relation logarithmique $V_{\lambda}=V+\lambda \operatorname{lnp}($ fig. $5 a)$. Il en résulte que, pour des murs hauts, de grandes valeurs de $\lambda$ produisent des valeurs de $\mathrm{K}_{p}$ plus petites, bien que proches, que dans le cas des petits $\lambda$. Dans ce cas, linfluence de la compressibilité existe toujours, mais est cachée par l'influence d'une grande plage de variation de contraintes dans la zone de rupture et par la courbure de l'enveloppe de rupture.
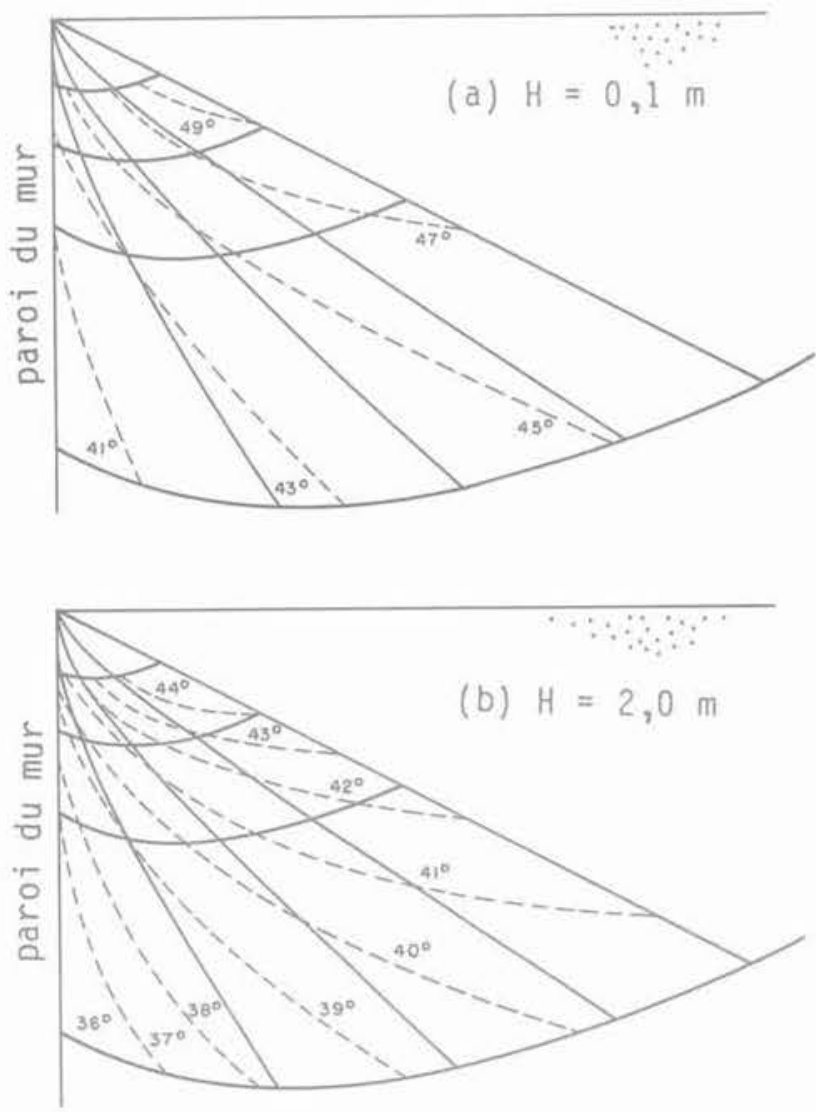

Fig. 11 - Valeurs de l'angle Ф localement mobilisé dans les zones de rupture de différentes dimensions.

Contrairement aux analyses habituelles du problème du mur de butée, le sable que nous considérons n'a pas une valeur unique de l'angle $\Phi$, prédéterminée par la densité de mise en place. La figure $4 \mathrm{~b}$ modélise un sable qui peut se dilater ou se comprimer lors du cisaillement, et qui localement peut mobiliser des valeurs $M(\Phi)$ différentes, fonction du niveau de contrainte. Ceci apparaît sur les figures $11 \mathrm{a}, \mathrm{b}$ où $\lambda=0,25$. La figure 11a est relative à une maquette de laboratoire avec $\mathrm{H}=0,1 \mathrm{~m}$, et la figure $11 \mathrm{~b}$ à un mur en vraie grandeur typique, avec $\mathrm{H}=2.0 \mathrm{~m}$. Les lignes pleines correspondent aux caractéristiques de contrainte qui décrivent la forme de la zone de rupture. A chaque intersection de deux caractéristi- ques de contrainte, les niveaux de contrainte sont connus et ainsi les valeurs de l'angle localement mobilisé $\Phi$ peuvent être évaluées. Les lignes en pointillé représentent pour la zone de rupture, les contours où la mobilisation de $\Phi$ est constante (iso- $\Phi$ ). La variation est de l'ordre de $8^{\circ}$ dans chacune des zones de rupture. Comme prévu, les valeurs de $\Phi$ sont plus grandes pour de petits murs que pour de hauts murs (de l'ordre de $5^{\circ}$ fig. $\left.11 \mathrm{a}, \mathrm{b}\right)$ et les valeurs de $\mathrm{K}_{p}$ suivent la même tendance. Dans ce cas, les valeurs sont $\mathrm{K}_{p}=25,5$ pour $\mathrm{H}=0,1 \mathrm{~m}$ et $\mathrm{K}_{p}=12,5$ pour $\mathrm{H}=2,0 \mathrm{~m}$.

\section{DISCUSSION}

Les résultats des figures 9 et 10 proviennent de l'utilisation d'un modèle de sol amélioré (le modèle d'état critique) dans une version avancée du programme qui utilise un angle $\Phi$ variable (comme prescrit par GRAHAM [1974]). Les lignes 1 et 2 de la figure 12 montrent les valeurs extrêmes atteintes dans l'analyse, respectivement par la plage de variation large avec $\lambda=0,25$ et par la plage de variation étroite avec $\lambda=0,05$. On montre donc que les relations $\mathrm{K}_{p}$ en fonction de $\log (\mathrm{H})$ sont similaires à celles obtenues par GRAHAM et POLLOCK (lignes 3 et 4 ) mais que, comme on peut s'y attendre. les résultats diffèrent dans le détail. On n'a pas essayé ici d'ajuster les données du sol de la figure 5 afin que les résultats des deux solutions coïncident. Toutefois, il est apparent que le nouveau modèle prédit des réductions substantielles de $\mathrm{K}_{p}$ quand $\mathrm{H}$ augmente (LAPER$\mathrm{CHE}, 1976)$, et prédit que les réductions dépendent de la compressibilité du sable.

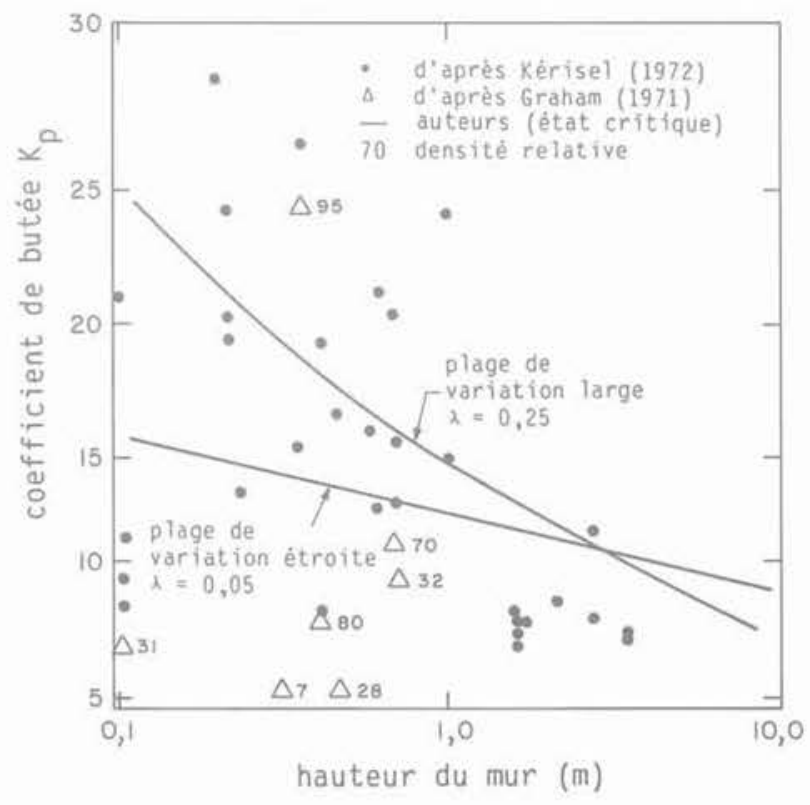

Fig. 12 - Comparaison des résultats de $K_{p}$ en fonction de lg (hauteur du mur). 
On peut comparer ces résultats avec des données disponibles de maquettes de laboratoire et de murs réels (fig. 13), dont les références sont fournies par GRAHAM (1971). Les données figurant dans les publications sont insuffisantes pour appliquer complètement le modèle d'état critique approprié à chaque programme expérimental. Toutefois, la tendance des données est claire, et va dans le sens des résultats des figures 9 et 10 .

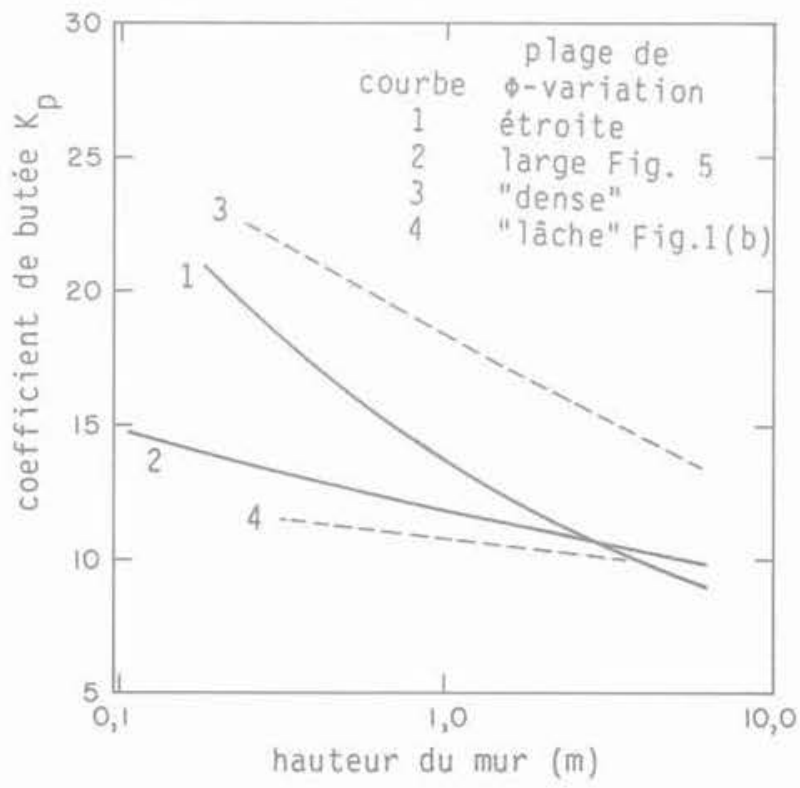

Fig. 13 - Comparaison des résultats théoriques de $K_{p}$ avec les données disponibles sur maquettes et sur murs réels.

Lorsque la hauteur du mur augmente, il y a changement évident du mécanisme de rupture : on passe d'une rupture générale de cisaillement le long de surfaces de rupture clairement définies avec petits mouvements, à une rupture par affaissement, où les phénomènes de compression du sol dominent et entraînent d'importants mouvements du mur. Au moins une partie de cette transition peut être attribuée au changement du comportement écrouissage-antiécrouissage qui accompagne, pour un sable, l'augmentation de la pression de confinement (fig. 2). Cet effet est inclus dans le modèle d'état critique de la figure $4 \mathrm{~b}$ et se répercute dans le calcul des valeurs $\mathrm{K}_{p}$.

Le point $\mathrm{B}$ de la figure 4 montre que lorsque les contraintes sont importantes (mais encore au-dessous des valeurs qui causent la fracture ou une déformation plastique des grains de sable), le modèle d'état critique définit une enveloppe de rupture qui approche une pente constante $\Phi_{m i n}$. A ce stade, on s'attend à des valeurs de $\mathrm{K}_{p}$ ou de $\mathrm{N}_{\gamma}$ plus ou moins constantes. Cet état n'est jamais atteint par des murs de butée, mais on doit le considérer pour les grandes plate-formes gravitaires de forage, où le coefficient $\mathrm{N}_{\gamma}$ peut atteindre une valeur constante minimale. L'analyse des semelles peu profondes a été récemment proposé par GRAHAM et HOVAN (1985). Lorsque les conditions de frontière d'un problème sont définies identiquement pour une solution d'équilibre limite et pour une solution résolue par la méthode des caractéristiques de contraintes, on trouve en principe la même valeur de la force de rupture (GRAHAM, 1974 ; SALENÇON, 1974). On doit donc se demander s'il y a quelque avantage associé à la complexité supplémentaire de la méthode des caractéristiques. Les données ci-dessus, et les articles précédents du deuxième auteur, ont montré que la méthode numérique des caractéristiques permet une définition plus précise des conditions de frontière (GRAHAM, 1971) et du comportement du sol (GRAHAM, 1974 ; GRAHAM et HOVAN, 1986). Par ces moyens, on obtient une modélisation plus générale du problème.

La question la plus fondamentale soulevée par cette étude est de savoir comment modéliser le comportement du sable. Traditionnellement, les comparaisons entre résultats théoriques et expérimentaux sont faites sur la base de a l'angle- $\Phi$ * du sable. Pourtant, il est reconnu que le sable n'a pas une valeur unique $\Phi$ et que des comparaisons ne peuvent être valables que si une valeur moyenne et appropriée pour la zone de rupture peut être évaluée (GRAHAM, 1971 ; HOVAN, 1985).

Si $\Phi$ est un paramètre inadéquat, que prendre en considération? L'application du modèle d'état critique de la figure $4 \mathrm{~b}$ demande peu d'essais supplémentaires, mais qui doivent être prévus dès la conception du programme d'essai ; celui-ci doit désormais inclure différentes densités de mise en place, différents niveaux de contrainte, et des mesures précises du changement de volume lors du cisaillement. Ce modèle de sol semble ouvrir d'importantes possibilités pour l'analyse du comportement des ouvrages. Ces possibilités n'ont pas encore été entièrement explorées, mais la présente étude montre qu'il peut rendre compte des effets d'échelle. Il faut toutefois noter que ce modèle de résistance de sol, relativement simple, n'a reçu qu'une validation partielle, et que des données d'essais supplémentaires sont nécessaires, relatives à une plus grande variété de sables de différentes minéralogies.

\section{CONCLUSIONS}

L'utilisation du modèle d'état critique dans la méthode des caractéristiques de contraintes fait apparaitre une relation plus ou moins linéaire entre $\mathrm{K}_{p}$ et log (hauteur du mur). La solution permet d'inclure l'effet du niveau de contrainte et de la densité de mise en place, ainsi que le comportement de plus en plus ductile qui est observé lorsque la hauteur du mur augmente. La tendance des résultats est conforme aux essais sur maquettes et sur murs réels. Des études supplémentaires sont nécessaires pour valider le modèle d'état critique à partir d'essais de résistance en laboratoire, et pour déterminer les paramètres relatifs à un grand nombre de sables différents.

\section{ANNEXE}

\section{NOTATION}

H Hauteur du mur

$\mathrm{K}_{p} \quad$ Coefficient du butée

M Rapport des contraintes $q / p$ à rupture 
$\mathrm{N}_{\gamma} \quad$ Coefficient de force portante des semelles pour l'effet des forces de poids propre dans la zone de rupture

p Contrainte effective moyenne $\left(\sigma_{1}+2 \sigma_{3}\right) / 3$

q Contrainte déviatorique $\left(\sigma_{1}-\sigma_{3}\right)$

$q_{u}, Q_{u}$ Contrainte (force) portante à rupture

$\mathrm{V}$ Volume spécifique, volume du sol occupé par volume unitaire des solides

$V_{\lambda} \quad V+\lambda \ln p$

$\gamma \quad$ Poids volumique du sol

$\lambda \quad$ Compressibilité du sable, pente de la droite normalement consolidée dans l'espace V, $\lg (\mathrm{p})$

$\mu \quad \pi / 4-\Phi / 2$

$\Phi \quad$ Angle de frottement

$\psi \quad$ Angle de $\sigma_{1}$ par rapport à l'axe z, positif dans le sens contraire des aiguilles d'une montre

$\sigma \quad$ (Sans indice) contrainte moyenne, $\left(\sigma_{1}+\sigma_{3}\right) / 2$, contrainte normale

$\tau \quad$ Contrainte de cisaillement

\section{INDICES}

$n, f$,

$\max$, min normal, rupture, maximale, minimale

$1,3, \mathrm{i}, \mathrm{0} \quad$ majeur mineur, $\mathrm{i}$-ième, initial

$\mathrm{tx}, \mathrm{ps}, \mathrm{v} \quad$ triaxial, en déformation plane, vertical

Tout au long du texte, les contraintes utilisées sont effectives.

\section{BIBLIOGRAPHIE}

1. AMAR, S. BAGUELIN F et CANEPA Y. (1984). Etude expérimentale du comportement des fondations superficielles, Sols et fondations, $n^{\circ} 427$ septembre 1984. Institut technique du bâtiment et des travaux publics, Paris, France.

2. ATKINSON J.H. et BRANSBY P.L. (1978), The mechanics of soils : an introduction to critical state soil mechanics, McGraw Hill (U.K.) Ltd., Maidenhead, England, $375 \mathrm{pp}$.

3. BEEN K. et JEFFERIES M.G. (1985), A state parameter for sands, Géotechnique, 35(2), pp. 99-112.

4. BIAREZ J., BOUCRANT L.-M. et NEGRE R. (1965), Equilibre limite d'écrans verticaux soumis à une translation ou une rotation, Comptes rendus $6^{e}$ Congrès International de Mécanique des Sols et des Travaux de Fondations, 2, pp. 368-372.

5. BJERRUM L. (1973), Geotechnical problems involved in foundations of structures in the North Sea, Géotechnique, 23(3), pp. 319-358.

6. DE BEER E.E. (1970), Experimental determination of the shape factors and the bearing capacity factors of sand, Géotechnique, London, England 20(4). pp. 387-411.

7. GRAHAM J. (1968), Plane plastic failure in cohesionless soils, Géotechnique, London, England, 18(3), pp. 301-316.

8. GRAHAM J. (1971), Calculation of passive pres- sure in sand, Revue canadienne de géotechnique, 8(4), pp. 566-578.

9. GRAHAM J. et POLLOCK D.J. (1972), Scale dependent plasticity analysis for sand, Civil Engineering and Public Works Review, London, England, 67, March 1972, pp. 245-251.

10. GRAHAM J. (1974), Plasticity solutions of stability problems in sand, Revue canadienne de géotechnique 11(4), mai 1974, pp. 238-247.

11. GRAHAM J., NOONAN M.L. et LEW K.V. (1983), Yield states and stress-strain relationships in a naturel plastic clay, Revue canadienne de géotechnique 20(3), pp. 502-516.

12. GRAHAM J. and HOVAN J.-M. (1986), Stress characteristics for bearing capacity in sand using a critcal state model, A paraître, Revue canadienne de géotechnique, 23(2).

13. HABIB P. (1985), Effet d'échelle et surfaces de glissement, Revue française de géotechnique, $\mathrm{n}^{\circ} 31$, pp. 5-10.

14. HAJAL M. (1961), Etude générale de la butée d'un écran plan contre un massif cohérent par la théorie des caractéristiques, Thèse, Laboratoire de Mécanique des Fluides, Université de Grenoble, France.

15. HOVAN J.-M. (1985), Computation of bearing capacity and passive pressure coefficients in sand using stress-characteristics and critical state, Thèse présentée à l'université de Manitoba, soutenue en février 1985, pour obtenir le diplôme de Maîtrise de Science.

16. JAMES R.G. et BRANSBY P.L. (1970), Experimental and theoretical investigations of a passive earth pressure problem, Géotechnique, 20(1), pp. 17-37.

17. KIMURA T., KUSAKABE O et SAITOH K (1985), Geotechnical model tests of bearing capacity problems in a centrifuge, Géotechnique, 35(1), pp. 33-46.

18. KRAVTCHENKO J, et SIBILLER. (1965), Etude des singularités rencontrées dans les problèmes d'équilibre limite, C.R. Acad. Sc., Paris, 261(2), pp. 2296 2298.

19. LADANYI B. (1960), Etude des relations entre les contraintes et les déformations lors du cisaillement des sols pulvérulents, Annales des Travaux Publics de Belgique, 3 , pp. 241-274.

20. LADE P.V. et LEE K.L. (1976), Engineering properties of soils, University of California-Los Angeles, Report UCLA-ENG-7652.

21. LAPERCHE J.P. (1976), Etude expérimentale de la force portante des fondations superficielles. Effet d'échelle et interaction. Thèse Institut de Mécanique, Grenoble.

22. MANDEL J. (1943), Equilibre par tranches planes des solides à la limite d'écoulement, Travaux, décembre 1943.

23. MORGENSTERN N.R. et EISENSTEIN Z. (1970), Methods of estimating lateral loads and deformations, ASCE Specialty Conference on Lateral Stresses in the Ground and Design of Earth Retaining Structures, Cornell University, pp. 51-102.

24. ROSCOE K.H. et BURLAND J.B. (1968), On the generalized stress-strain behaviour of "wet" clay, From "Engineering Plasticity ». Cambridge University Press, pp. 535-609. 
25. ROWE P.W. (1969), Progressive failure and strengh of a sand mass, Proc. 7th International Conference on Soil Mechanics and Foundation Engineering, Mexico, 1, pp. 341-349.

26. SALENÇON J. (1972), Butée d'une paroi lisse sur un massif plastique solutions statiques, J. Mécanique, 11(1), pp. 135-146.

27. SALENÇON J. (1974), Théorie de la plasticité pour les applications à la mécanique des sols, Editions Eyrolles, Paris, 1974.

28. SALENÇON J., MANDEL J. et GABRIEL Y. (1976), Capacité portante globale d'une fondation sur un sol non-homogène, Géotechnique, 26(2), pp. $351-370$.
29. SOKOLOVSKII V.V. (1965), Statics of granula: media, Pergamon Press, New York, N.Y.

30. TCHENG Y, et ABSI E. (1969), Poussée et butée en vraie grandeur, Comptes rendus du 7 e Congrès lnternational de Mécanique des Sols et des Travaux des Fondations.

31. VESIC A.S. (1973), Analysis of ultimate loads of shallow foundations, ASCE Journal of the Soil Mechanics and Foundations Division, 99 (1), pp. 45-73.

32. VESIC A.S et CLOUGH G.W. (1968), Behaviour of granular material under high stresses, ASCE, SMFE Division, 94(3), pp. 661-688. 